\title{
Anderson-Fabry: una malattia rara?
}

\author{
Paolo Colomba, Giuseppe Cammarata, Carmela Zizzo, Simone Scalia, Daniele Francofonte, Riccardo Alessandro, \\ Emanuela Marsana, Lidia Consoli, Serena Guttadauria, Francesco lemolo, Vincenzo Savica, Giovanni Duro
}

Istituto di Biomedicina e Immunologia Molecolare "A. Monroy", Consiglio Nazionale delle Ricerche, Palermo

\begin{abstract}
Anderson-Fabry: a rare disease?
Anderson-Fabry (or Fabry) disease takes the name from the two dermatologists that independently described for the first time the pathology at the end of 1800. It is a multisystemic lysosomal storage disease, inherited in a X-linked manner. Fabry manifestations are slowly progressive, with variable age of onset, severity and clinical course. Usually, in the childhood or in the first period of the adolescence, the patients can manifest angiokeratomas, corneal or lenticular opacity, microalbuminuria or proteinuria and symptoms reflecting the involvement of peripheral nervous system, like episodic crises of acute pain, acroparesthesias, anhidrosis or hypohidrosis. With the age, there is a progression of the disease with a gradual deterioration of kidney function, that leads to End Stage Renal Disease (ESRD), and the onset of severe cardiovascular and cerebrovascular complications, that cause a reduction in quality of life in affected patients. Such complications, or a combination of these, can lead to the premature death of the patient within the fourth or fifth decade of life. In addition to the classic form, atypical variants of the disease were described: these variants manifest a mild and late-onset clinical phenotype, that is difficult to diagnose because of the involvement of a single organ. The $80 \%$ of Fabry patients are affected by these variants with a non-specific symptomatology that makes the diagnosis, which can be made in the adulthood after several years since the manifestation of the first symptoms, when the internal organs are irreversibly compromised, difficult. It was estimated a considerable delay in the diagnosis in about $40 \%$ of male patients and $70 \%$ of female patients. In particular, there is a time interval of 13 years for men and 17 years for women between the onset of the first symptoms and the correct diagnosis. Therefore, Fabry disease should be considered among the diagnostic hypothesis in all the subjects that present a systemic clinical course referable to the disease. The availability of recombinant enzyme replacement therapy has an important impact on clinical management of affected patients, improving the prognosis and the quality of life. The data in literature show the importance of a specific therapeutic intervention made as early as possible, before an irreversible organ involvement.
\end{abstract}

Keywords: Anderson-Fabry disease, $\alpha$-galactosidase A, GLA gene, Misdiagnosis

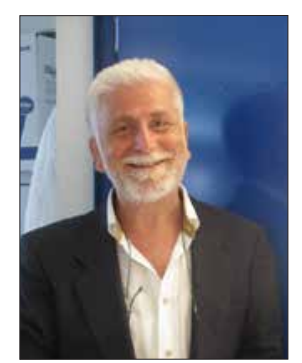

Giovanni Duro

\section{Malattia di Anderson-Fabry}

È un disordine metabolico, da accumulo lisosomiale, caratterizzato dal deficit funzionale dell'enzima $\alpha$-galattosidasi A ( $\alpha-G A L$ A) (1). Tale deficit determina un'alterazione del metabolismo di alcuni glicosfingolipidi, prevalentemente globotriaosilceramide (Gb3) e Lyso-GB3, che, di conseguenza, si accumulano nei

\section{Accepted: May 7, 2015}

Published online: June 4, 2015

Indirizzo per la corrispondenza:

Prof. Giovanni Duro

Istituto di Biomedicina e Immunologia Molecolare "A. Monroy"

Consiglio Nazionale delle Ricerche

Via Ugo La Malfa 153

90146 Palermo

giovanni.duro@ibim.cnr.it lisosomi di numerosi tipi cellulari, soprattutto delle cellule dell'endotelio vascolare (2). La malattia è considerata rara, con un'incidenza stimata intorno a 1:40.000 nella popolazione generale, ma, in recenti iniziative di screening neonatale, è stata riscontrata in 1:3.100 neonati maschi in Italia (3) e in 1:1.500 neonati maschi in Taiwan (4). Dal punto di vista eziologico, la malattia di Fabry è un'enzimopatia lisosomiale determinata da mutazioni nel gene GLA, che codifica per l' $\alpha$-GAL A, situato nel braccio lungo del cromosoma $X$ in regione q21-22 (5). Tale localizzazione determina una trasmissione della patologia di tipo $X$-linked; ciò significa che un soggetto maschio affetto può trasmettere il difetto solo alla prole di sesso femminile, mentre una donna affetta ha il $\mathbf{5 0} \%$ di possibilità di trasmetterlo sia alla prole di sesso maschile che a quella femminile. A oggi, sono state descritte più di 770 mutazioni nelle regioni codificanti di GLA (Human Gene Mutation Database, www.hgmd.org), in pazienti Fabry, senza, tuttavia, riuscire a stabilire una chiara corrispondenza tra genotipo e fenotipo. II quadro clinico classico della malattia di Fabry insorge generalmente già in età pediatrica con acroparestesie urenti all'estremità degli arti (6). La 
compromissione del sistema nervoso autonomo è responsabile dell'alterazione della sudorazione, che diviene un elemento critico in particolari situazioni, come la febbre e lo sforzo fisico, momenti in cui, per il disordine della termoregolazione, si possono avere delle tipiche crisi di dolore. Anche le manifestazioni dolorose gastrointestinali, associate a diarrea alternata a senso di pienezza addominale, trovano spiegazione nel coinvolgimento del sistema vegetativo locale. La cute nella malattia di Fabry può manifestare i tipici angiocheratomi espressi principalmente nelle aree della "mutandina" o, addirittura, un angiocheratoma corporis diffusum e la presenza di un linfedema soprattutto degli arti inferiori. L'occhio, come in molte malattie neuro-metaboliche, è interessato, con espressioni che vanno dalla tipica cornea verticillata alla cataratta e alle teleangiectasie della congiuntiva. L’accumulo di glicosfingolipidi nelle cellule renali ne compromette la funzione, portando a una nefropatia progressiva che è una delle caratteristiche più critiche della malattia. In uno studio europeo, la prevalenza della malattia di Fabry tra gli individui maschi dializzati è risultata essere dello $0.264 \%$ (7). Nei pazienti Fabry, il cuore presenta frequentemente un'ipertrofia ventricolare sinistra, apparentemente idiopatica, e una diretta compromissione dell'apparato valvolare e della conduzione intracardiaca, con complicanze che vanno dall'insufficienza cardiaca alla patologia aritmica, che può portare a morte per infarto del miocardio. II sistema endocrino è stato oggetto di indagini approfondite e frequentemente presenta deficit legati alle caratteristiche di elevata vascolarizzazione e al basso turnover cellulare propri del tessuto endocrino. Oltre alla compromissione del sistema nervoso periferico, il paziente con malattia di Fabry presenta un coinvolgimento diretto dell'encefalo con stroke ischemici giovanili e possibili episodi emorragici. Tuttavia, il quadro clinico classico, completo di tutte le manifestazioni tipiche della malattia, viene riscontrato solamente in circa il $20 \%$ dei pazienti affetti. L' $80 \%$ di essi, invece, presenta varianti atipiche della malattia, caratterizzate da un fenotipo clinico lieve, a esordio tardivo, e frequentemente con il coinvolgimento di un solo organo. Inoltre, i pazienti di sesso femminile, in seguito al fenomeno della lyonizzazione, presentano generalmente una sintomatologia più sfumata e variabile rispetto a quelli di sesso maschile. II sospetto di Fabry viene avanzato in base ai dati clinici e a quelli anamnestico-familiari e, infine, viene confermato attraverso il dosaggio dell'attività dell' $\alpha$ galattosidasi $A$, che può essere nulla o deficitaria, e mediante I'indagine molecolare con ricerca di mutazioni causali nel gene GLA. Anche la determinazione dei substrati dell'enzima (Gb3 e Lyso-Gb3), accumulati nei lisosomi delle cellule di diversi organi e tessuti, potrebbe fornire un supporto diagnostico.

\section{Malattia di Fabry in pazienti con disturbi renali}

I disturbi renali sono tra le principali manifestazioni cliniche della malattia di Fabry che, come la maggior parte dei sintomi tipici della malattia, peggiorano con l'età. La nefropatia nella malattia di Fabry esordisce clinicamente con la comparsa di microalbuminuria e proteinuria che caratterizza il 50\% dei pazienti di sesso maschile oltre i 35 anni e il $\mathbf{1 0 0 \%}$ dei pazienti oltre i $\mathbf{5 0}$ anni. Superata la quinta decade di vita non è rara I'insufficienza renale terminale. Approssimativamente il 30$35 \%$ delle donne adulte affette da malattia di Fabry manifesta proteinuria, il $13 \%$ presenta una malattia renale cronica allo stadio 3, mentre una piccola percentuale (1-4\%) raggiunge l'insufficienza renale terminale (8-11). Nella malattia di Fabry è possibile osservare una vasta gamma di quadri istopatologici renali (12). Infatti possono essere interessati tutti i tipi cellulari renali anche in pazienti con GFR normale e minima proteinuria (13), anche se la vacuolizzazione dei podociti e delle cellule epiteliali rimane la caratteristica principale della malattia di Fabry $(12,13)$. A livello cellulare, nei podociti, il progressivo accumulo di Gb3 porterebbe ad alterazioni cellulari e a un danno istologico. La disfunzione podocitaria causerebbe, conseguentemente, un'alterazione della barriera di filtrazione glomerulare e proteinuria. A livello tissutale, la deposizione di Gb3 può provocare danni di natura ischemica, che sono il risultato della sofferenza endoteliale microvascolare e della necrosi delle cellule muscolari lisce vascolari. Sempre a livello delle cellule muscolari lisce vascolari, il Gb3 ne può promuovere la proliferazione e il rilascio di mediatori coinvolti nelle nefropatie (14). Un altro fattore da considerare è rappresentato dai danni causati dall'infiammazione locale conseguente alla rottura dei lisosomi $(15,16)$. Uno studio giapponese ha identificato la malattia di Fabry nell' $1.2 \%$ dei pazienti di sesso maschile con insufficienza renale terminale, che avevano precedentemente ricevuto una diagnosi di glomerulonefrite cronica (17). La maggior parte di questi pazienti (83\%) non presentava manifestazioni cliniche classiche che avrebbero agevolato la diagnosi di malattia di Fabry, suggerendo che essa potrebbe essere sottodiagnosticata tra i pazienti sottoposti a dialisi renale e/o trapianto. Nella malattia di Fabry, l'insufficienza renale è una delle principali cause di morte, infatti aumenta considerevolmente il rischio di eventi cardiovascolari, e, conseguentemente, i pazienti Fabry che sviluppano malattia renale all'ultimo stadio corrono un maggiore rischio di sperimentare eventi cardiovascolari e ictus rispetto agli altri pazienti con malattia di Fabry in genere. L'intervento precoce con ERT, se utilizzata come parte di una strategia globale per prevenire le complicanze della malattia di Fabry, può contribuire a stabilizzare la funzione renale o a rallentare il suo declino. Anche se i dati sull'efficacia della ERT sono basati su pochi studi controllati a lungo termine, la ERT ha dimostrato la capacità di limitare il calo della funzione renale sia in pazienti di sesso maschile che in quelli di sesso femminile così come in pazienti pediatrici (18).

\section{Una malattia spesso vista ma raramente diagnosticata}

La malattia di Fabry è una patologia considerata rara ma, in realtà, come emerge dalla recente letteratura, sarebbe più giusto considerarla un disordine non comune, poco conosciuto. La diagnosi risulta ancora oggi difficile proprio per le 
peculiarità della malattia, che si presenta con manifestazioni cliniche sovrapponibili a quelle di altre patologie e un'ampia possibilità di diagnosi differenziali che coinvolgono diverse specializzazioni mediche (19). Le difficoltà nel diagnosticare questa patologia sono evidenziate da studi retrospettivi che hanno rilevato un ritardo considerevole nella diagnosi in circa il $40 \%$ dei pazienti di sesso maschile e nel $70 \%$ dei pazienti di sesso femminile. In particolare, dall'insorgenza dei primi sintomi alla corretta diagnosi trascorrono, mediamente, 13 anni per gli uomini e 17 per le donne. Questi dati confermerebbero le considerazioni di Hoffmann e Mayatepek, secondo cui la malattia di Fabry è un disordine spesso visto ma raramente diagnosticato (20).

Dal momento che l'espressione clinica della malattia di Fabry può essere facilmente confusa con altre patologie sistemiche, nei nostri laboratori abbiamo condotto uno studio su pazienti i cui sintomi e le cui origini geografiche avevano portato alla diagnosi clinica di Febbre Mediterranea Familiare (FMF), un disordine reumatico con alcune manifestazioni sovrapponibili a quelle della malattia di Fabry. Su 42 soggetti analizzati, il $7.2 \%$ presentava mutazioni nel gene GLA associate alla malattia di Fabry: in alcuni casi si è trattato di concomitanza delle due malattie, mentre, in altri casi, di errore diagnostico (21).

Questi risultati confermano che l'errore diagnostico è un rischio concreto e determina una sottostima del reale numero di soggetti affetti. Studi su altre patologie con possibili casi di Fabry misconosciuta/misdiagnosticata sono attualmente in corso nei nostri laboratori.

\section{Fenomeno della lyonizzazione nella malattia di Fabry}

In quanto patologia legata al cromosoma X, la malattia di Fabry può comportare delle differenze nelle manifestazioni cliniche tra uomini e donne. Infatti, gli individui di sesso femminile, di solito eterozigoti, i cui organi sono chimere di cellule normali e malate a causa dell'inattivazione di uno dei due cromosomi X (lyonizzazione), hanno generalmente sintomi meno evidenti e, quindi, di più difficile individuazione. Fino a qualche anno fa le donne venivano considerate portatrici; questo concetto è stato rivisto e oggi è noto che anche la donna può presentare manifestazioni gravi della malattia. Dal momento che anche le donne eterozigoti possono sviluppare un danno d'organo irreversibile, un attento follow-up clinico deve essere effettuato a prescindere dai loro valori di attività enzimatica.

\section{La nostra esperienza}

Dal 2005, presso il Laboratorio di Biotecnologie Applicate alle Scienze Mediche dell'Istituto di Biomedicina e Immunologia Molecolare del CNR di Palermo, il nostro gruppo di ricerca si dedica allo studio delle alterazioni enzimatiche e genetiche nella malattia di Fabry. In questi anni abbiamo eseguito i saggi,

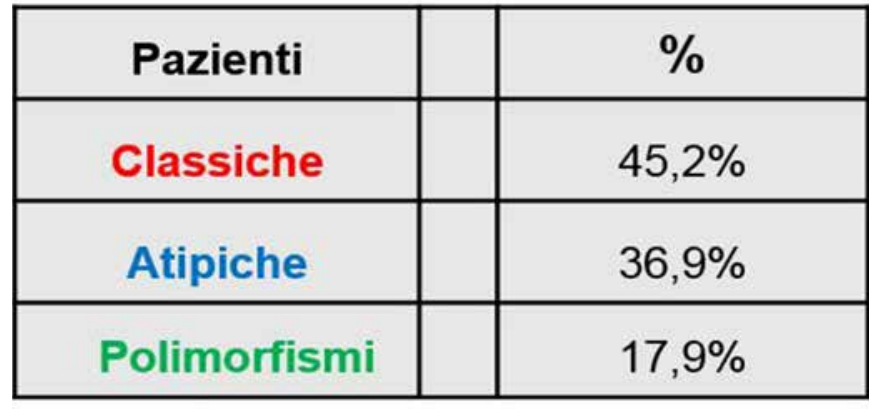

Fig. 1 - Mutazioni esoniche nel gene GLA, riscontrate nel nostro studio, associate a fenotipo classico, a varianti atipiche e polimorfismi.

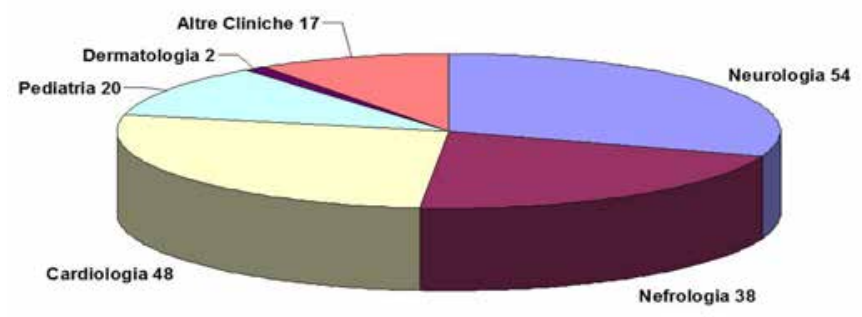

Fig. 2 - Distribuzione delle 179 mutazioni esoniche, da noi riscontrate, nelle specializzazioni cliniche di provenienza. I dati sono riferiti al periodo 2005-2015.

genetici ed enzimatici, su circa 8000 soggetti, con segni e sintomi riconducibili alla malattia, pervenuti da diverse cliniche distribuite sull'intero territorio nazionale, e 3000 controlli.

Nel nostro studio, di case-finding, abbiamo riscontrato la presenza di mutazioni nelle regioni codificanti del gene GLA in 179 dei soggetti analizzati. La maggior parte di tali mutazioni è riportata nelle banche dati come responsabile della malattia di Fabry, mentre altre sono state da noi descritte per la prima volta nel mondo e, integrando i dati genetici con quelli biochimici e clinici, sono state associate alla malattia (22-24).

È importante sottolineare che, soltanto per una piccola percentuale dei pazienti affetti, in cui l'indagine genetica ha confermato la malattia, era stata formulata tempestivamente I'ipotesi diagnostica di Fabry, mentre la maggior parte di essi aveva, in precedenza, ricevuto altre diagnosi e, pertanto, si trattava di casi misconosciuti/misdiagnosticati.

Le mutazioni esoniche da noi identificate possono essere catalogate in tre diversi gruppi: associate al fenotipo classico, a varianti atipiche e alleli pseudodeficienti non responsabili della malattia di Fabry, secondo la letteratura (Figs. 1, 2).

\section{Attività enzimatica}

Dal punto di vista dell'analisi enzimatica, il Dried Blood Filter Paper (DBFP) test, un metodo comunemente usato per la determinazione dell'attività dell' $\alpha$-galattosidasi $A$ nel sangue, nel nostro laboratorio, è stato ulteriormente migliora- 
to e ottimizzato. I nostri dati mostrano che, nella totalità dei soggetti maschi con mutazioni nel gene GLA, associate alla malattia di Fabry, l'attività dell' $\alpha$-galattosidasi A è inferiore ai valori normali, confermando la validità del metodo. La stessa corrispondenza non si osserva nelle pazienti di sesso femminile dove, a causa del fenomeno dell'inattivazione di uno dei due cromosomi $X$, nelle diverse cellule dell'organismo, l'attività enzimatica è estremamente variabile oscillando tra valori normali e patologici (25). Un nostro studio retrospettivo ha mostrato che più del $57 \%$ dei pazienti di sesso femminile affetti dalla malattia di Fabry presentava valori di attività enzimatica del tutto nella norma. Pertanto, nelle donne, l'analisi genetica costituisce l'unico mezzo per confermare la diagnosi clinica di malattia di Fabry.

\section{Conclusioni}

La malattia di Fabry si può presentare in maniera estremamente eterogenea, anche all'interno di uno stesso gruppo familiare. Nella sua forma classica, la malattia si manifesta sin dall'infanzia con sintomatologia neurologica periferica, per coinvolgere poi progressivamente, con l'avanzare dell'età, il rene, il cuore e il sistema nervoso centrale. Questo processo degenerativo può assumere varie forme ed esprimersi attraverso diverse sfumature: l'età di insorgenza, i segni e i sintomi e la loro gravità. In alcuni casi, si è assistito al coinvolgimento di un unico organo, mentre, altre volte, la malattia si manifesta solamente con una fastidiosa sintomatologia dolorosa e con un minimo coinvolgimento d'organo (rene, cuore, cervello).

Nel complesso, sulla base della nostra esperienza, i pazienti che presentano manifestazioni cliniche riferibili alla malattia di Fabry possono essere raggruppati in tre categorie:

a) soggetti con la forma classica della malattia e mutazioni nel gene GLA, che codifica l' $\alpha$-galattosidasi A;

b) soggetti con varianti atipiche della malattia (forma lieve ed esordio tardivo) e mutazioni nel gene GLA, diverse da quelle responsabili della forma classica;

c) soggetti con sintomi riconducibili alla malattia di Fabry e privi di mutazioni nelle regioni codificanti del gene GLA.

Nei primi due gruppi, la dimostrazione della presenza di una mutazione nelle regioni codificanti del gene GLA fornisce una conferma della diagnosi clinica avanzata dal medico. Invece, nei soggetti che presentano i sintomi della malattia di Fabry ma che sono privi di mutazioni in GLA, circa il $98 \%$ dei soggetti che giungono alla nostra attenzione, si rende necessario l'utilizzo di strumenti diagnostici innovativi. La scoperta di nuovi marcatori diagnostici è di fondamentale importanza sia per aiutare nella diagnosi di Fabry sia per migliorare le conoscenze cliniche e molecolari in merito alla patogenesi della malattia. Nel nostro laboratorio sono attualmente in corso dei progetti che mirano a individuare potenziali marcatori molecolari della malattia di Fabry.
Dal punto di vista clinico, la malattia di Fabry dovrebbe essere sempre presa in considerazione quando giungono all'osservazione pazienti con decorsi clinici atipici, diagnosi incerte o quadri clinici con coinvolgimento sistemico non chiari, soprattutto in presenza di crisi dolorose non giustificate, parestesie, intolleranza al freddo o al caldo, ipoidrosi, angiocheratomi, crampi addominali e cornea verticillata. In soggetti adulti in cui vengono rilevati ictus criptogenetico, cardiomiopatia ipertrofica o danno renale manifestato con proteinuria, possono essere sospettate la forma classica o possibili varianti atipiche della malattia. Anche lo studio della storia clinica familiare può indirizzare il clinico, tenendo conto della trasmissione della patologia legata al cromosoma $X$.

Pensando alla malattia di Fabry, è importante sottolineare che non bisogna aspettarsi di riscontrare contemporaneamente tutte le manifestazioni cliniche presenti nella forma classica, ma si deve considerare che l' $80 \%$ dei pazienti è affetto da varianti atipiche con sintomi sfumati, generalmente a esordio tardivo e con il coinvolgimento di un solo organo.

Sono stati elaborati diversi algoritmi diagnostici per guidare i clinici verso la diagnosi di malattia di Fabry e, nei soggetti in cui viene avanzata l'ipotesi diagnostica, la conferma può venire dalle analisi genetiche e biochimiche. Anche la determinazione dell'accumulo di Gb3 e Lyso-Gb3 può confermare la diagnosi clinica.

Una diagnosi precisa e tempestiva è essenziale per avviare precocemente i pazienti alla terapia enzimatica sostitutiva, che è in grado di rallentare o arrestare la progressione della malattia, migliorandone, così, la qualità della vita.

\section{Disclosures}

Financial support: No financial support was received for this submission.

Conflict of interest: The authors have no conflict of interest.

\section{Bibliografia}

1. Brady RO, Gal AE, Bradley RM, Martensson E, Warshaw AL, Laster L. Enzymatic defect in Fabry's disease. Ceramidetrihexosidase deficiency. N Engl J Med. 1967;276(21):1163-7.

2. Desnick RJ, loannou YA, Eng CM. Alpha-galactosidase A deficiency: Fabry disease. In: Scriver CR, Beaudet AL, Sly WS, Valle $\mathrm{D}$, editors. The metabolic and molecular basis of inherited disease. 8th ed. New York: McGraw-Hill. 2001:3733-74.

3. Spada M, Pagliardini S, Yasuda M, et al. High incidence of lateronset fabry disease revealed by newborn screening. Am J Hum Genet. 2006;79(1):31-40.

4. Hwu WL, Chien YH, Lee NC, et al. Newborn screening for Fabry disease in Taiwan reveals a high incidence of the later-onset GLA mutation c.936+919G>A (IVS4+919G>A). Hum Mutat. 2009;30(10):1397-405.

5. Kornreich R, Bishop DF, Desnick RJ. The gene encoding alphagalactosidase $A$ and gene rearrangements causing Fabry disease. Trans Assoc Am Physicians. 1989;102:30-43.

6. Germain DP. Fabry disease. Orphanet J Rare Dis. 2010;5:30.

7. Kotanko P, Kramar R, Devrnja D, et al. Results of a nationwide screening for Anderson-Fabry disease among dialysis patients. 
J Am Soc Nephrol. 2004;15(5):1323-9.

8. Wilcox WR, Oliveira JP, Hopkin RJ, et al. Females with Fabry disease frequently have major organ involvement: lessons from the Fabry Registry. Mol Genet Metab. 2008;93(2):112-28.

9. Ortiz A, Oliveira JP, Waldek S, et al. Nephropathy in males and females with Fabry disease: cross-sectional description of patients before treatment with enzyme replacement therapy. Nephrol Dial Transplant. 2008;23(5):1600-7.

10. Weidemann F, Niemann M, Sommer C, Beer M, Breunig F, Wanner $C$. Interdisciplinary approach towards female patients with Fabry disease. Eur J Clin Invest. 2012;42(4):455-62.

11. Ortiz A, Cianciaruso B, Cizmarik M, et al. End-stage renal disease in patients with Fabry disease: natural history data from the Fabry Registry. Nephrol Dial Transplant. 2010;25(3):769-75.

12. Utsumi K, Mitsuhashi F, Asahi K, et al. Enzyme replacement therapy for Fabry disease: morphologic and histochemical changes in the urinary sediments. Clin Chim Acta. 2005;360(1-2): 103-7.

13. Selvarajah M, Nicholls K, Hewitson TD, Becker GJ. Targeted urine microscopy in Anderson-Fabry disease: a cheap, sensitive and specific diagnostic technique. Nephrol Dial Transplant. 2011;26(10):3195-202.

14. Sanchez-Niño MD, Sanz AB, Carrasco S, et al. GlobotriaosyIsphingosine actions on human glomerular podocytes: implications for Fabry nephropathy. Nephrol Dial Transplant. 2011;26(6):1797-802.

15. Valbuena C, Carvalho E, Bustorff M, et al. Kidney biopsy findings in heterozygous Fabry disease females with early nephropathy. Virchows Arch. 2008;453(4):329-38.

16. Safyan R, Whybra C, BeckM, Elstein D, Altarescu G. An association study of inflammatory cytokine gene polymorphisms in Fabry disease. Eur Cytokine Netw. 2006;17(4):271-5.

17. Nakao S, Kodama C, Takenaka T, et al. Fabry disease: detection of undiagnosed hemodialysis patients and identification of a "renal variant" phenotype. Kidney Int. 2003;64(3):801-7.

18. Germain DP, Charrow J, Desnick RJ, et al. Ten-year outcome of enzyme replacement therapy with agalsidase beta in patients with Fabry disease. J Med Genet. 2015;52(5): 353-8.

19. Marchesoni CL, Roa N, Pardal AM, et al. Misdiagnosis in Fabry disease. J Pediatr. 2010;156(5):828-31.

20. Hoffmann B, Mayatepek E. Fabry disease-often seen, rarely diagnosed. Dtsch Arztebl Int. 2009;106(26):440.

21. Zizzo C, Colomba P, Albeggiani G, et al. Misdiagnosis of familial Mediterranean fever in patients with Anderson-Fabry disease. Clin Genet. 2013;83(6):576-81.

22. Colomba P, Nucera A, Zizzo C, et al. Identification of a novel mutation in the alpha-galactosidase $A$ gene in patients with Fabry disease. Clin Biochem. 2012;45(10-11):839-41.

23. Tuttolomondo A, Duro G, Miceli $S$, et al. Novel alphagalactosidase $A$ mutation in a female with recurrent strokes. Clin Biochem. 2012;45(16-17):1525-30.

24. Duro G, Musumeci MB, Colomba P, et al. Novel $\alpha$-galactosidase A mutation in patients with severe cardiac manifestations of Fabry disease. Gene. 2014;535(2):365-9.

25. Dobrovolny R, Dvorakova L, Ledvinova J, et al. Relationship between X-inactivation and clinical involvement in Fabry heterozygotes. Eleven novel mutations in the alpha-galactosidase A gene in the Czech and Slovak population. J Mol Med. 2005; 83(8):647-54. 